\title{
MIMO Multi Carrier Interleave Division Multiple Access System with Multiuser Detection: Performance Results
}

\author{
Prabagarane Nagaradjane $^{1}$, Sai N. Chandrasekaran ${ }^{2}$, Kuttathatti Srinivasan Vishvaksenan ${ }^{3}$ \\ ${ }^{1}$ Department of Electronics and Communication Engineering, SSN College of Engineering, \\ SSN Institutions, Chennai, India \\ ${ }^{2}$ The University of Kansas, Kansas, USA \\ ${ }^{3}$ Department of ECE, SSN College of Engineering, SSN Institutions, Chennai, India \\ Email:prabagaranen@ssn.edu.in,csnivi@gmail.com,vishvaksenanks@ssn.edu.in \\ Received January 6, 2010; revised February 17, 2010; accepted March 16, 2010
}

\begin{abstract}
This paper provides the performance analysis of multi input multi output (MIMO) Multi carrier (MC) interleaver based multiple-access system with multiuser detection. Interleave-division multiple access (IDMA) is a scheme in which users are separated by employing different interleavers instead of different signatures as in a conventional code-division multiple-access (CDMA) scheme. The basic principle of IDMA is that the interleaver is different for different users. Interleavers are generated independently and randomly. At the receiver, ZF, LLSE, VBLAST/ZF/MAP and VBLAST/LLSE/MAP detectors are employed. The performance of the system is analyzed for different channel conditions using extensive simulation runs based on Monte Carlo simulation trials. Simulation results divulge that VBLAST/LLSE/MAP detector results in significant performance amelioration compared to other detectors as applied to MC/IDMA system.
\end{abstract}

Keywords: CDMA, Channel Capacity, Iterative Decoding, Multi-User Detection

\section{Introduction}

This paper presents an approach to asynchronous multiple access scheme called interleave division multipleaccess (IDMA) [1] system with MIMO support. In an IDMA scheme, different interleavers are used to distinguish users as against different codes in a conventional code division multiple access (CDMA) system. In a conventional CDMA scheme, interleavers are placed before the spreaders and they are effective only when used in conjunction with channel coding [2,3]. Recently, a very interesting technique using chip-level interleavers was addressed in [1], which aims at mitigating intersymbol interference (ISI) in multipath fading environments. Many papers have discussed the role of interleavers in a multiple access systems [1,3]. IDMA inherits many benefits of CDMA; in particular, path diversity and mitigation of intra cell interference. Also all the users employ a common spreading sequence. Recent research on wireless communication systems has shown that using multiple antennas at both transmitter and receiver offers the possibility of communications at higher data rates compared to single antenna systems [4-6]. Multi input and multi output (MIMO) system has proved in the recent past to provide very high capacity without any increase in the transmission bandwidth and power. The information-theoretic capacity of these multiple-input multiple-output channels was shown to grow linearly with smaller numbers of transmit and receive antennas in rich scattering environments, and at sufficiently high signalto-noise (SNR) ratios [7-9]. MIMO profile in any wireless communication system can be realized by two schemes namely, 1) using the classical BELL labs architecture (VBLAST-Vertical Bell Laboratories Space Time architecture) and 2) Space Time Block Codes. And OFDM techniques can compensate for multi-path fading effects, which cause time dispersion, and out of band emission of the received signal. So combining these techniques with the IDMA system can result in MIMO MC/IDMA that can offer bandwidth efficiency, space diversity and lower speed parallel type of signal processing and interference rejection capability (ISI reduction) in high data-rate transmission. Of late, significant progress has been made in multi-user detection for codedivision multiple-access (CDMA) systems [6]. In this paper, we investigate the performance of the MIMO as- 
sisted Multi Carrier interleave division multiple access (MIMO MC/IDMA) scheme with multiuser detection. We will show that the MIMO MC/IDMA scheme can provide better performance with the aid of VBLAST/ LLSE/MAP [5,6] detection technique. Also this approa$\mathrm{ch}$ is independent of the number of users and gives better performance in multipath environment. The rest of the paper is organized as follows - the system model is presented in Section 2. Section 3 gives the gist of signal detection techniques. Performance results will be presented in Section 4. Eventually conclusions are drawn in Section 5 .

\section{System Model}

In this section, we introduce the IDMA scheme with Multi carrier modulation. The block diagram of MIMO MC/IDMA scheme is elucidated in Figure 1. In IDMA the interleavers are different for different users. Interleavers are generated independently and randomly. Different interleavers separate the users and all the users employ a common spreading sequence. Interleavers, which are usually placed between forward error correction (FEC) coding and spreading, is used to combat fading effect in CDMA, whereas the arrangement of interleaving and spreading is reversed in IDMA. Now, different interleavers distinguish distinct data streams. In IDMA, encoder block may do FEC encoding and spreading jointly. The spreader has no special function. In this paper we consider a single user MIMO MC IDMA system. Each of the synchronous user is equipped with $M$ transmit antennas and they communicate to a single base station, equipped with $N$ receive antennas. MIMO profile is realized by using VBLAST (Vertical Bell Laboratories
Space Time architecture).

\section{Symbol Detection Techniques}

Considering a synchronous signal over a time variant channel, the received signal at the time instant $j$ is given as

$$
r(j)=\sum_{k=1}^{\min (m, n)} \sum_{k}^{k} H_{k} x_{k}(j)+n(j), j=1,2, \ldots, J
$$

where $H_{k}$ is the channel co-efficient for user $k$ and $\{n(j)\}$ are samples of an AWGN process with variance $\sigma^{2}=$ $N o / 2$. Here perfect knowledge of the channel state information is assumed to be available at the receiver. In each use of the MIMO channel, a vector $x=\left(x_{1}, x_{2} \ldots x_{M}\right)^{T}$ of complex numbers is sent and a vector $r=\left(r_{1}, r_{2}, \ldots\right.$, $\left.r_{N}\right)^{T}$ of complex numbers is received. We assume an input-output relationship of the form

$$
r=H x+n
$$

where $H$ is a $N \times M$ matrix $(N=$ number of transmit antennas and $M=$ number of receive antennas) representing the scattering effects of the channel and $n=\left(n_{1}\right.$, $\left.n_{2}, \ldots, n_{N}\right)^{T}$ is the noise vector. Throughout, we assume that $H$ is a random matrix with independent complex Gaussian elements $\left\{h_{i j}\right\}$ with mean 0 and unit variance, denoted by $h_{i j} \sim N(0,1)$. We also assume throughout that $n$ is i.i.d complex Gaussian random vector. The symbol detection problem considered in this paper is the problem of estimating the MIMO channel input vector $x$ given the received vector $r$ assuming that the receiver has perfect knowledge of $H$. The decision is made on a symbol-by-symbol basis. The next section discusses the various VBLAST detectors employed in our work.

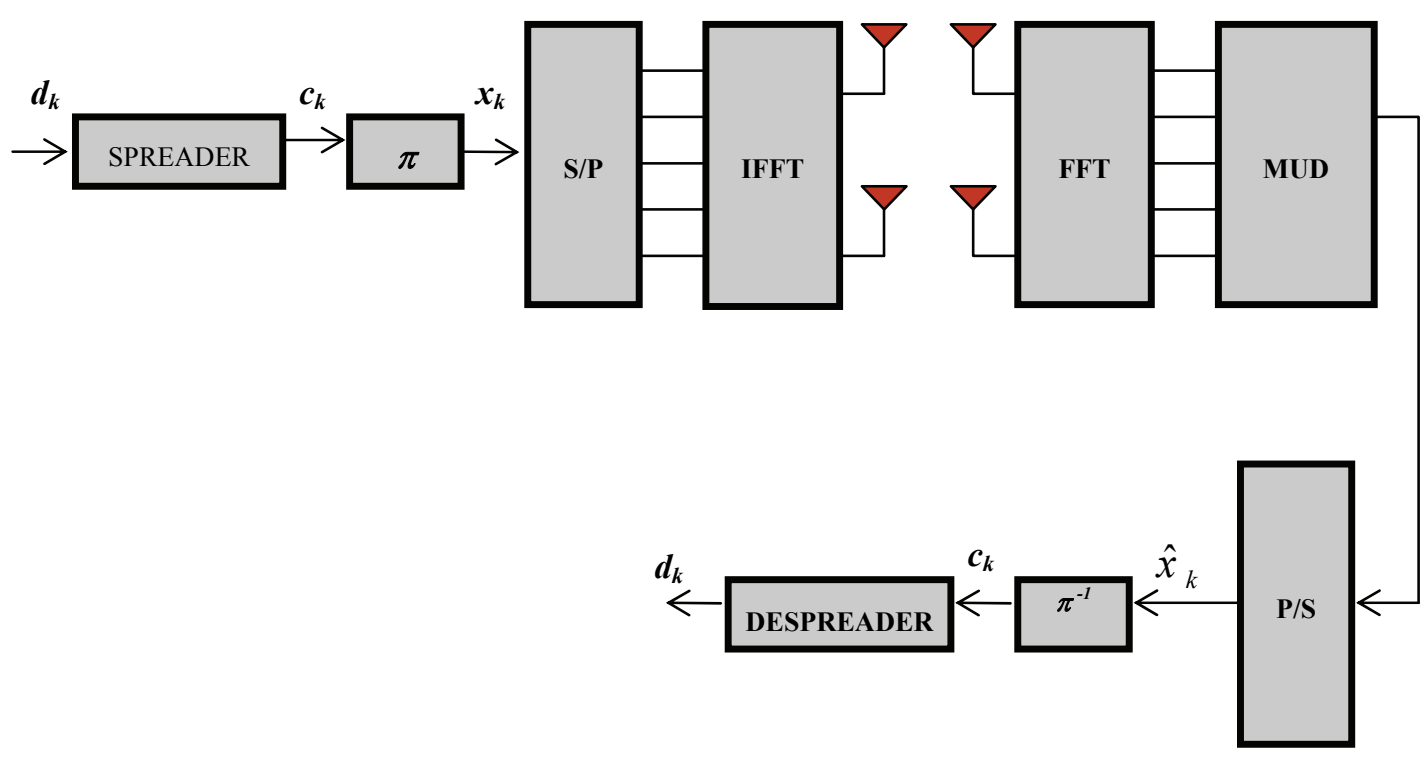

Figure 1. MIMO MC/IDMA system. 


\subsection{Zero-Forcing (ZF) Receiver}

Zero-forcing (ZF) receiver is a low-complexity linear detection algorithm that outputs,

$$
\hat{x}=Q\left(\hat{x}_{Z F}\right)
$$

where

$$
\hat{x}_{Z F}=H^{+} \quad r
$$

and $H^{+}$denotes the Moore-Penrose pseudo inverse of $H$, which is a generalized inverse that exists even when $H$ is rank-deficient.

\subsection{Linear Least Square Error Receiver (LLSE)}

LLSE receiver is also a linear detection algorithm that outputs

$$
\hat{a}=Q\left(\hat{a}_{L L S E}\right)
$$

where

$$
\hat{a}_{L L S E}=W r
$$

and when $H$ and $n$ are gaussian, $W$ is the weight vector given by

$$
W=\frac{\rho}{M} H^{+}\left(\frac{\rho}{M} H H^{+}+N_{o} I_{N}\right)^{-1}
$$

\subsection{V-BLAST Detection}

The V-BLAST detection algorithm [6] is a recursive procedure that extracts the components of the transmitted vector $\boldsymbol{x}$ according to a certain ordering $\left(k_{1}, k_{2}, \ldots, k_{M}\right)$ of the elements of $\boldsymbol{x}$, where, $\left(k_{1}, k_{2}, \ldots, k_{M}\right)$ is a permutation of $(1, \ldots, M)$. In VBLAST, this permutation depends on $\boldsymbol{H}$ (which is known at the receiver by assumption) but not on the received vector $\boldsymbol{r}$. The VBLAST/ZF algorithm is a variant of VBLAST derived from ZF rule. The algorithm determines the order of channels to be detected, performs nulling and computes the decision statistics. It then slices the computed decision statistics and yields the decision by performing cancellation with the help of decision feedback, and finally computes the new pseudo-inverse for the next iteration. V-BLAST/ZF may be seen as a successive-cancellation scheme derived from the $\mathrm{ZF}$ scheme discussed in the previous section. The ZF rule creates a set of subchannels by forming

$$
\hat{x}=\left(H^{+} H\right) x+H^{+} n
$$

The $j^{\prime}$ th such sub-channel has noise variance $\left\|\left(\mathrm{H}^{+}\right)_{j}\right\|^{2} N_{0}$ and the order selection rule prioritizes the sub-channel with the smallest noise variance.

\subsection{VBLAST/MAP Detection Algorithm}

In this section, a detection algorithm for MIMO channels, called VBLAST/MAP that combines the features of
VBLAST and MAP rules is used for symbol detection in a MC/IDMA system. This algorithm does not use the layered structure of VBLAST, but uses a different strategy for channel processing order, inspired by the MAP rule. This algorithm is an extension of the well-known VBLAST algorithm. This VBLAST/MAP combines the elements of VBLAST algorithm and the maximum a-posteriori (MAP) rule. The original VBLAST algorithm is a multilayer symbol detection scheme which detects symbols transmitted at different transmit antennas successively in a certain data-independent order. The VBLAST/ MAP algorithm differs from VBLAST only in the ordering strategy of the symbols detected. The complexity of the VBLAST/ MAP is higher than that of VBLAST; however, the performance improvement is significant.

\subsection{V-BLAST/ZF/MAP Detection Algorithm}

Using the same notation of VBLAST algorithm, VBLAST/ZF/MAP algorithm may be described, as followsVBLAST/ZF/MAP algorithm is identical to VBLAST/ ZF except for the ordering in which symbols are detected. Instead of selecting the next symbol to be detected according to the rule, here the set of all probable symbol decisions are ranked with respect to their $a$-posteriori probabilities of being correct, as estimated by $p_{i j}$. Thus, it is important to emphasize that $p_{i j}$ 's are not true MAP probabilities but approximations to how probable it is that $s_{i j}$ $=x_{j}$. Note that the index permutation $\left(k_{1}, k_{2}, \ldots, k_{M}\right)$ produced by VBLAST/ZF/MAP depends on both $H$ and $r$, unlike VBLAST/ZF where the permutation depends only on $\boldsymbol{H}$. Complexity of VBLAST/ZF/MAP is increased with respect to that of VBLAST/ZF by the computation steps involved. One major point about complexities of VBLAST/ZF and VBLAST/ZF/MAP is that, the former allows pre-computation of all weighting vectors whereas in the latter the weighting vector must be computed in real-time since it also depends on $\boldsymbol{r}$. This increased complexity of VBLAST/ZF/MAP is justified by performance improvements. The algorithm is summarized as follows:

\section{Initialization}

$$
\begin{gathered}
W_{1}=H^{+} \\
i=1
\end{gathered}
$$

Recursion

$$
\begin{aligned}
& y_{i}=W_{i} r_{i} \\
& s_{i}=Q\left(y_{i}\right) \\
& p_{i j}=\frac{f_{i j}\left(y_{i j} \mid s_{i j}\right)}{\sum_{s^{\prime} \in A} f_{i j}\left(y_{i j} \mid s^{\prime}\right)}, j \notin\left\{k_{1} \ldots k_{i-1}\right\} \\
& k_{i}=\underset{j \notin\left\{k_{1} \ldots k_{i-1}\right\}}{\arg \max }\left\{p_{i j}\right\} \\
& \hat{x}_{k_{i}}=S_{i} k_{i}
\end{aligned}
$$




$$
\begin{aligned}
& r_{i+1}=r_{i}-\hat{x}_{k_{i}}(H)_{k_{i}} \\
& W_{i+1}=H_{\bar{k}_{i}}^{+} \\
& i=i+1
\end{aligned}
$$

\subsection{V-BLAST/LLSE/MAP Detection Algorithm}

This detection algorithm is akin to V-BLAST/ZF/MAP detection except the weight vector defined by the Equation (7).

\section{Performance Results}

Figure 2 expounds the simulation results for an $8 \times 12$ MIMO MC/IDMA system employing ZF, LLSE, VBLAST/ZF and VBLAST/LLSE detectors. The modulation technique considered is $16-\mathrm{QAM}$ and the $E_{b} / N_{0}$ ranges between $-10 \mathrm{~dB}$ and $4 \mathrm{~dB}$. The symbol error rate SER is

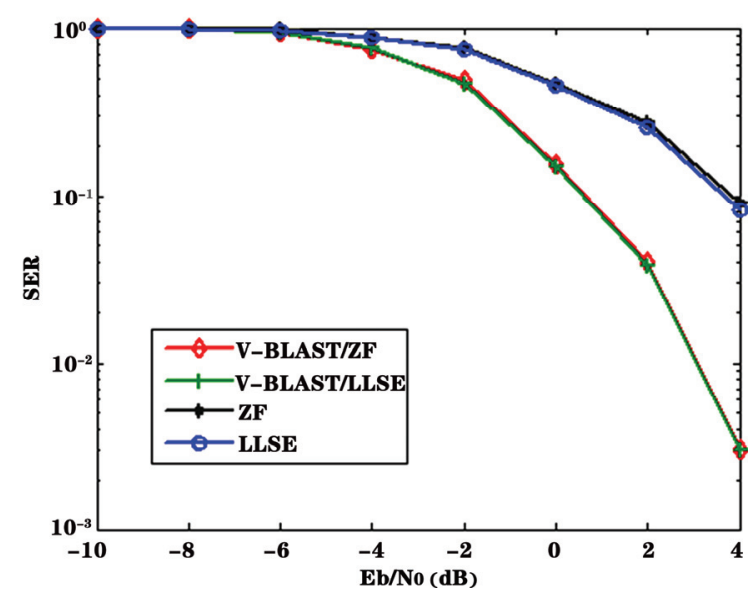

Figure 2. Symbol error rates (SER) of VBLAST/ZF, VBLAST/LLSE, ZF and LLSE receiver.

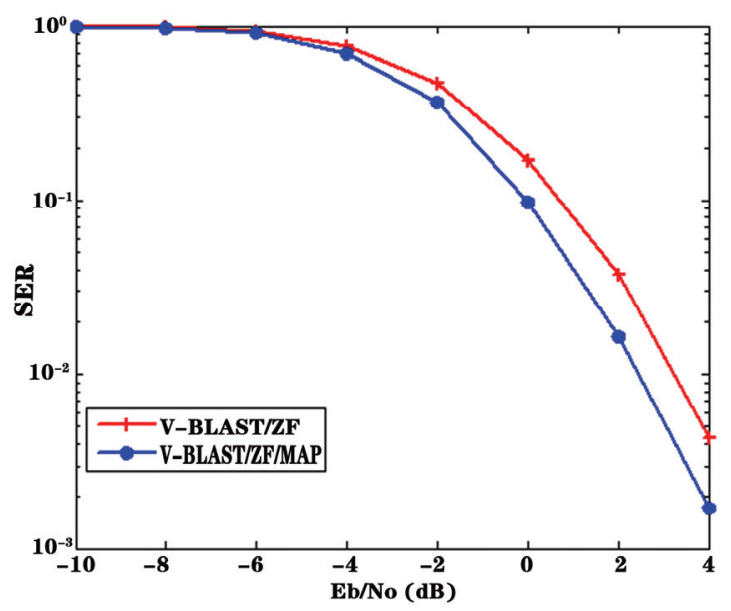

Figure 3. Symbol error rates (SER) of VBLAST/ZF/MAP and VBLAST/ZF receiver.

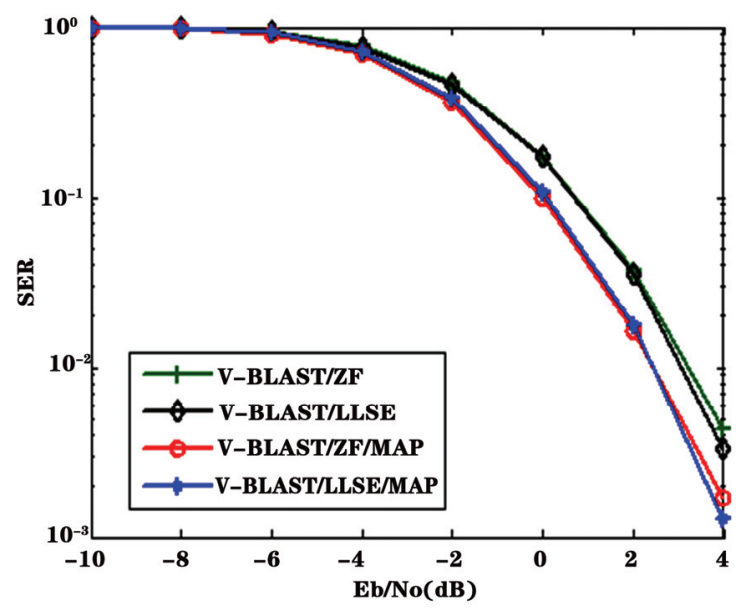

Figure 4. Symbol error rates (SER) of VBLAST/ZF, VBLAST/LLSE, VBLAST/ZF/MAP and VBLAST/LLSE/MAP receiver.

calculated by performing 10,000 trials at each $E_{b} / N_{0}$ point. A new realization of $\boldsymbol{H}$ was chosen in each trial and for each $E_{b} / N_{0}$ value. The performance curves in Figure 2 show that VBLAST/LLSE provides significant improvement in SER compared to ordinary VBLAST/ZF, ZF and LLSE detector. Figure 3 elucidates the simulation results for an $8 \times 12$ MIMO MC/IDMA system employing VBLAST/ZF and VBLAST/MAP detectors. It is discerned that VBLAST/MAP outperforms VBLAST/ZF detector. Figure 4 evinces the simulation results for an $8 \times$ 12 MIMO MC/IDMA system employing VBLAST/ZF, VBLAST/LLSE, VBLAST/ZF/MAP and VBLAST/LLSE /MAP receiver with 16QAM modulation. It is discerned that VBLAST/LLSE/MAP outperforms VBLAST/ZF/ MAP detector in terms of SER performance.

\section{Conclusions}

In this paper a multiple access scheme for $4 \mathrm{G}$ wireless communications has been considered based on interleavers. Problems in two major areas have been identified in this paper: efficient transmission technique and symbol detection in the presence of multi stream interference. A solution has been conceived to achieve the above. Simulation results have proved that the MIMO MC/IDMA in combination with VBLAST/LLSE/MAP multiuser detector can achieve significant performance improvement compared to ZF, LLSE, and VBLAST/ZF/MAP.

\section{References}

[1] H. Schoeneich and P. A. Hoeher, "Adaptive InterleaveDivision Multiple Access - A Potential Air Interface for 4G Bearer Services and Wireless LANs," International Conference on Communications, 2004.

[2] C. S. Nivedita, S. Padmini and C. Vijay, "Interleaver 
Based Multiple Access System: A Proposal for 4G Mobile Communication with Iterative Chip-by-Chip MultiUser Detection," Project Report, SSN Institutions, 2006.

[3] N. Prabagarane, C. S. Nivedita, S. Padmini and C. Vijay, "Interleave Division Multiple Access System with Iterative Multiuser Detection," IEEE International Conference and Exposition on Advanced Ceramics and Composites, February 2007.

[4] N. Prabagarane, M. Ramakrishnan, T. Divya, M. Thomas and B. Jalaja, "Performance Evaluation of Multi Stage Receivers for Coded Signals in MIMO Channels," 4th IEEE WiCOM, October 2008.

[5] T. Divya, M. Thomas and B. Jalaja, "Performance Evaluation of Multi Stage Receivers for Coded Signals in MIMO Channels," Project Report, SSN Institutions, 2008.

[6] V. Arun, A. S. S. Vasan and L. Krishnan, "Investigations on the Performance of MIMO Assisted Multi Carrier DS/CDMA System with Multiuser Detection for 4G Mobile Communications," Project Report, SSN Institutions, 2009.

[7] I. E. Telatar, "Capacity of Multi-Antenna Gaussian Channels," Europe Transportation and Telecommunication, Vol. 10, No. 6, November-December 1999, pp. 585-595.

[8] P. Nagaradjane, A. S. S. Vasan and L. Krishnan, "A Robust Space Time Co-Channel Interference Mitigation and Detection Technique for Multiuser MIMO Multicarrier DS/CDMA Systems," Proceedings of IEEE International Conference on Wireless Vitae, Montreal, 2009.

[9] P. Nagaradjane, A. S. S. Vasan, L. Krishnan and A. Venkataswamy "Joint VBL-AST/STBC Assisted MC DS/CDMA System with Multiuser Detection," Proceedings of IEEE International Conference, WiCOM, October 2009. 